\title{
Supplementary Materials: On-the-Fly Femtosecond Action Spectroscopy of Charged Cyanine Dyes: Electronic Structure versus Geometry
}

\author{
Marius Hervél, Richard Brédy*1, Gabriel Karras ${ }^{1}$, Bruno Concina ${ }^{1}$, Jeffery M. Brown², \\ Abdul-Rahman Allouche ${ }^{1}$, Franck Lépine ${ }^{1}$, and Isabelle Compagnon ${ }^{1,3}$
}

${ }^{1}$ Univ Lyon, Université Claude Bernard Lyon 1, CNRS, Institut Lumière Matière, F-69622, Villeurbanne, France

${ }^{2}$ Waters Corporation, Stamford Avenue, Altrincham Road, Wilmslow SK9 4AX, United Kingdom

${ }^{3}$ Institut Universitaire de France IUF, 103 Boulevard St. Michel, Paris F-75005, France

\author{
Corresponding Author \\ *richard.bredy@univ-lyon1.fr
}

\section{Multiple-Photon Femtosecond Action Spectroscopy of On-the-fly Molecular Ions}

The experimental results were obtained on our new experimental setup, combining a highrepetition rate tunable femtosecond laser with a triple quadrupole instrument. This allows for performing action spectroscopy without any trapping device, in a cross-beam configuration, despite the non-negligible velocity of the ions in the linear quadrupoles, necessary for well-guiding conditions. Figure S1 compares the Collision-Induced Dissociation (CID) mass spectrum obtained for [IR797] ${ }^{+}$in typical conditions of the instrument (Fig S1.a) with a mass spectrum obtained after interaction with the laser, thus without any gas in the collision cell (Fig S1.b). The same main fragments (methyl and $\mathrm{HCl}$ losses) are found in both cases, with different branching ratios since it depends on the acceleration voltages in the case of CID. This suggests that the fragmentation induced by photo-absorption is of statistical nature as in CID, and doesn't come from direct dissociation processes from the $S_{1}$ excited state.

Figure S1.c presents a power dependence of the photofragmentation yield of [IR797 $]^{+}$at $\lambda_{\max }=760 \mathrm{~nm}$. The non-linear evolution is characterized in log-log scale by a slope of 2.6 \pm 0.1 . This slope is due to the fact that some fragments show a non-linearity of 3 , while others show a non-linearity of 2 . Therefore, at least two photons are necessary to observe fragmentation in our experiment. Given the low energy per pulse (up to $50 \mathrm{~nJ} /$ pulse) and the 
absence of resonant excited states around $400 \mathrm{~nm}$ in the studied species, we believe that this two-photon process is sequential. A first photon is absorbed to the excited state, which relaxes back to the ground state by transferring its energy to the vibrations by Intramolecular Vibrational energy Redistribution (IVR). The molecule can then absorb a second photon to the same excited state, which relaxes again towards the vibrational degrees of freedom, providing enough energy to statistically fragment. This is possible in our experiment due to the very high repetition rate $(80 \mathrm{MHz})$ of the laser: the ions stay long enough in the laser focus to interact with several laser shots, each of them being separated by $12.5 \mathrm{~ns}$. This is also supported by comparing the typical $\mathrm{C}-\mathrm{C}$ and $\mathrm{C}-\mathrm{Cl}$ bond energies (around 3-4 eV) with the photon energy (1.6 eV at $\lambda=760 \mathrm{~nm}$ ).
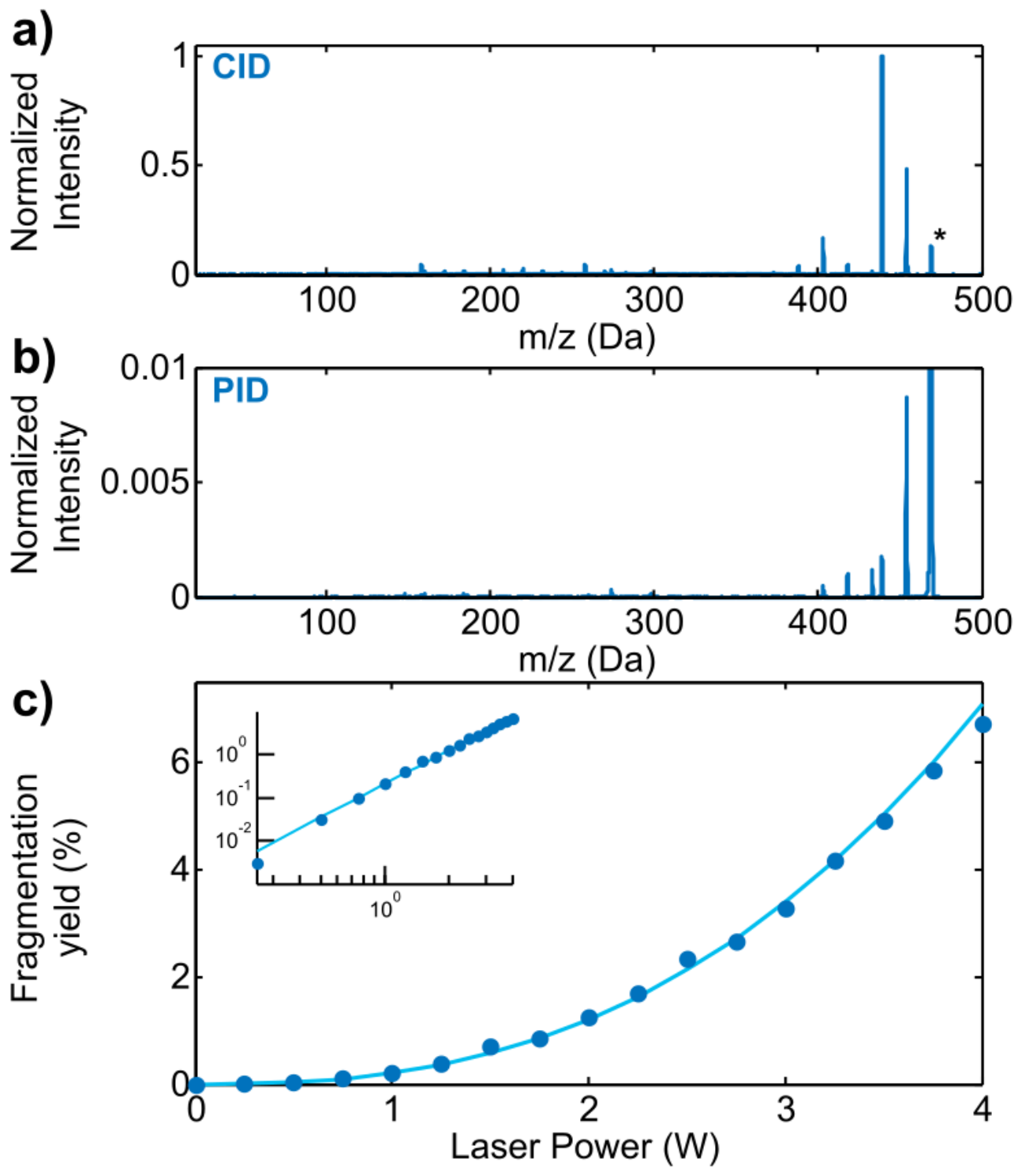

Figure S1. a) CID mass spectrum of $[I R 797]^{+}$, for a collision energy of $30 \mathrm{~V}$ and an argon pressure of $4,0.10^{-3}$ mbar (the ${ }^{*}$ marker indicates the parent ion). b) Photofragmentation mass spectrum, for $\lambda=760 \mathrm{~nm}, \mathrm{P}=2.0 \mathrm{~W}$. Both CID and PID spectra are normalized on the maximum of each mass spectrum. c) Fragmentation yield at $\lambda=760 \mathrm{~nm}$ as a function of the laser power (blue dots). The light blue curve corresponds to a power-law fit, yielding a 2.6 \pm 0.1 exponent, extracted from a linear fit of the log-log curve (inset). 
Cyanine molecules are known to aggregate in solution ${ }^{1}$. We also observed such cyanine aggregates in the gas phase. Figure S2.b shows singly charged aggregates of two IR806 molecules with a single proton $\left([2 \mathrm{IR} 806.3 \mathrm{H}]^{+}, \mathrm{m} / \mathrm{z}=1425\right)$. However, no doubly charged dimers (as for example [2IR806.4H $]^{2+}$ ) were observed in our experiments, as confirmed by the isotopic distribution (mass separation of $1 \mathrm{Da}$ ) of the [IR806.2H] ${ }^{+}$monomer (Fig S1.a).
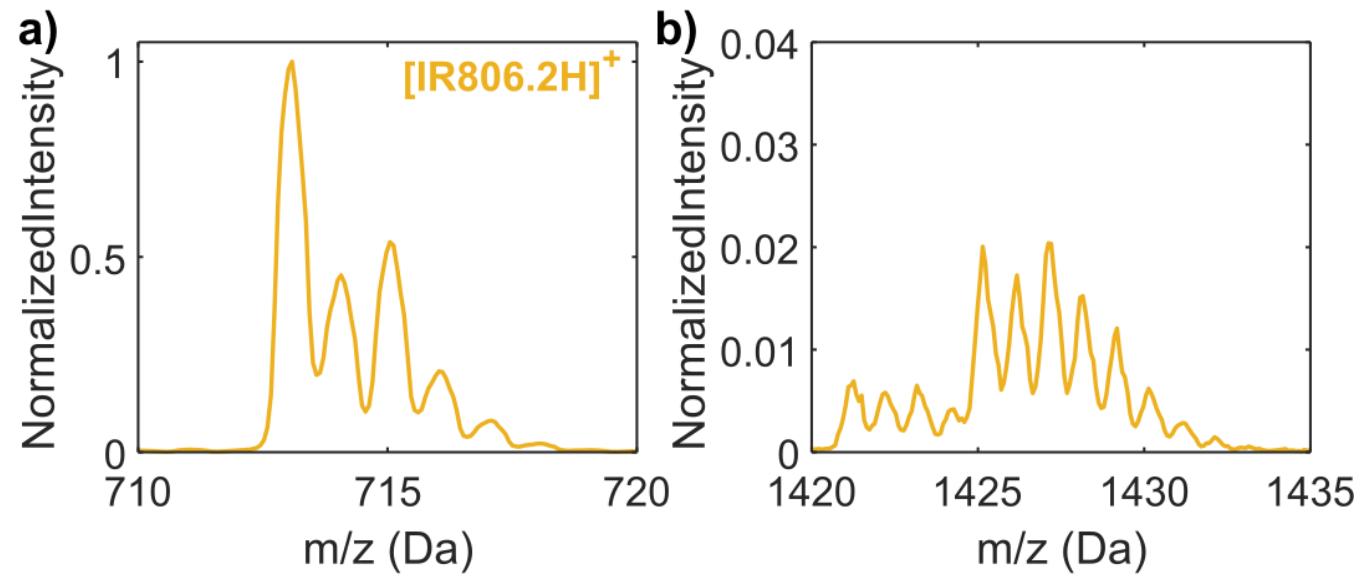

Figure S2. a) Isotopic distribution of $[\mathrm{IR} 806.2 \mathrm{H}]^{+}(\mathrm{m} / \mathrm{z}=713)$, recorded without selection in mass by the first quadrupole. b) Mass spectrum centered around $m / z=1425$, showing the presence of a singly charged dimer $[2 \mathrm{IR} 806.3 \mathrm{H}]^{+}$along with its isotopic distribution.

\section{Ground State Geometry of the Gas Phase Cyanines}

As described in the Computational Methods section in the main text, geometries were first optimized by TDDFT calculations using the B3LYP functional at the $6-31+G *$ level. We first performed calculations on the different possible configurations, depending whether the nitrogen substituents are pointing towards the same side of the molecule or not, and towards the same side as the chlorine atom. Figure S3 displays the optimized planar geometries in the three considered configurations, together with their energy, for [IR806]'. The most stable configuration corresponds to the geometry where both sulfonated substituents lie on the same side of the molecule, but opposite to the chlorine atom (called Syn1 thereafter). It is interesting to also note that the absorption wavelength does not change significantly with the different configurations, due to very small changes for the polymethine chromophore. We thus chose the Syn1 configuration for all the further calculations of the substituted IR806 derivatives and for [IR797] ${ }^{+}$. 

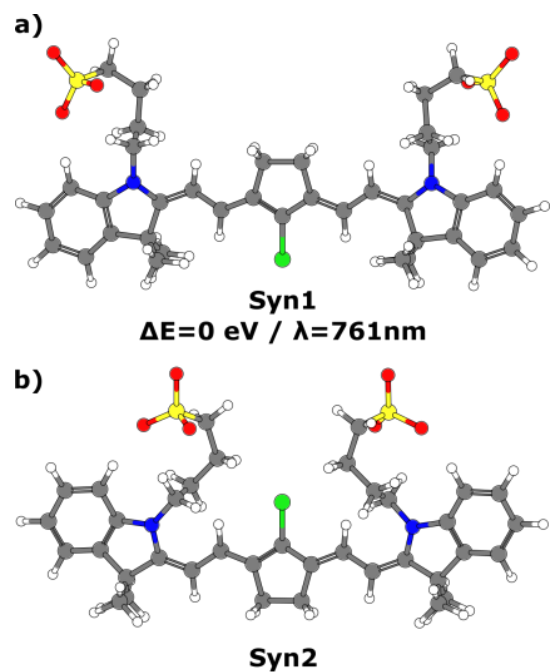

$\Delta E=+279 \mathrm{meV} / \lambda=766 \mathrm{~nm}$

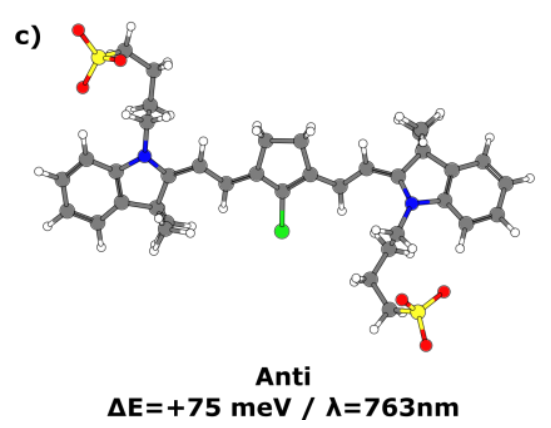

Figure S3. Optimized geometries for the three configurations considered here: a) the Syn1 configuration, with both sulfonated substituents on the same side of the polymethine chain but at the opposite of the chlorine atom (most stable configuration); the Syn2 configuration, with sulfonated substituents and the chlorine atom on the same side of the polymethine chain; c) the Anti configuration, with the sulfonated substituents on opposite sides of the polymethine chain. The B3LYP/6-31+G* energy difference relative to the Syn1 configuration is calculated for the configurations, and the calculated $S_{0}-S_{1}$ transition wavelengths (at the $\mathrm{CIS}(\mathrm{D}) /$ def2-tzvp level of theory) are also given for each configuration.

Conformational exploration was then performed starting from the Syn1 configuration for the different species. This was done for [IR806] $]^{-}$using MMF94 exploration, and local optimization was then performed after addition of counter cations for the positively charged IR806 derivatives. Figure S4 shows the result of the conformational study for [IR806] ${ }^{-}$and [IR806.2Na] ${ }^{+}$. In the case of [IR806]', the most stable conformation corresponds to the geometry with sulfonated substituents curving back to the indole groups, which is $1.09 \mathrm{eV}$ more stable than the conformation with "straight" substituents. For [IR806.2Na] ${ }^{+}$, it is found that a conformation where the two sulfonated substituents fold toward the center of the molecule, complexing the two $\mathrm{Na}^{+}$cations, is more stable than conformations with straight arms or arms curved back to the indole groups by $1.37 \mathrm{eV}$. This is also the case for the other IR806 cationic derivatives. 
a) $[\mathrm{IR806}]^{-}$

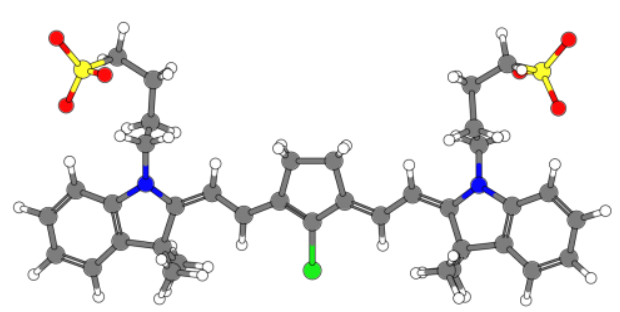

$\Delta E=0$ eV / $\lambda=761 \mathrm{~nm}$

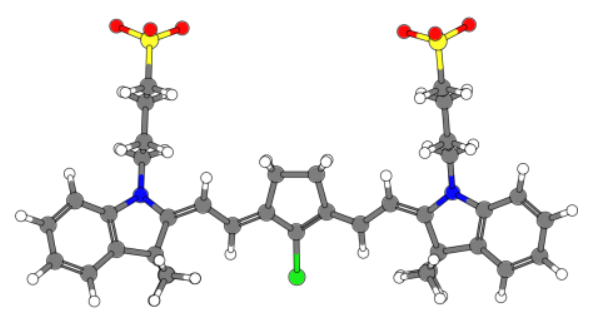

$\Delta E=+1.09 \mathrm{eV} / \lambda=756 \mathrm{~nm}$

b) $[\text { IR806.2Na }]^{+}$

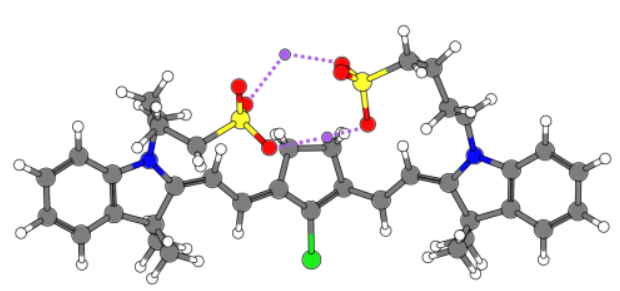

$\Delta E=0$ eV / $\lambda=778 \mathrm{~nm}$

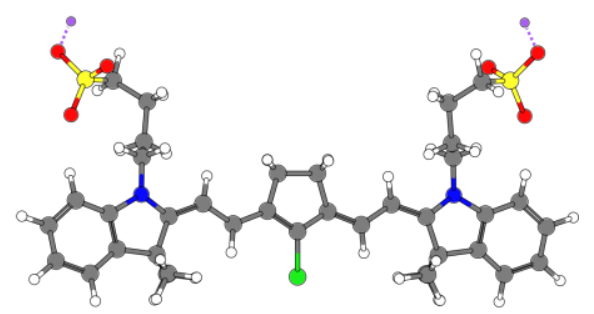

$\Delta E=+1.37 \mathrm{eV} / \lambda=757 \mathrm{~nm}$

Figure S4. Geometries of different considered conformations, for [IR806] ${ }^{-}$(a) and $\left[I R 806.2 \mathrm{Na}^{+}\right.$(b), together with their energy difference relative to the most stable conformation (left) and the computed $\mathrm{S}_{0}-\mathrm{S}_{1}$ transition wavelength. For [IR806]', the most stable conformation and a conformation with "straight" nitrogen substituents are displayed. For [IR806.2Na] $]^{+}$, the most stable (folded) conformation and a conformation with unfolded nitrogen substituents are displayed. Relative energies are calculated using the B3LYP/6$31+G^{*}$ method for [IR806] ${ }^{-}$and the MP2/def2-tzvp method for [IR806.2Na] ${ }^{+}$(since this method was used to compute the charges), while the wavelengths are calculated using the CIS(D)/def2-tzvp one.

Figure S5 summarizes the different most stable structures of the five studied species, obtained in our calculations. As discussed in the main text, [IR806. HNa] ${ }^{+}$exists in two conformations, with similar energies $(\triangle E(D F T)=+141 \mathrm{meV}$, while $\Delta E(M P 2)=-113 \mathrm{meV})$. For the cationic derivatives, the side view shows that the induced folding results in a bending of the polymethine chromophore. The dihedral angle is also shown for these species, measured as the angle around the central $\mathrm{C}-\mathrm{Cl}$ axis between the carbon atoms labeled (-4) and (+4) (see Fig $\mathrm{S} 6$ for atom labels attribution). 

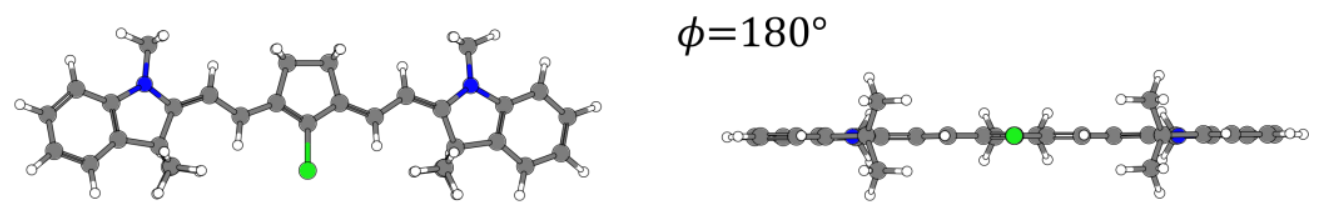

b) $[$ IR806]-: $761 \mathrm{~nm}$
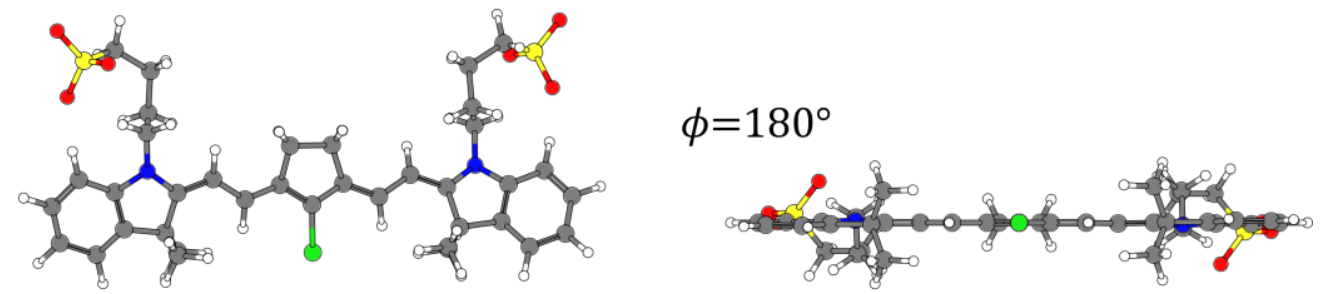

c) $[\text { IR806.2H }]^{+}: 760 \mathrm{~nm}$
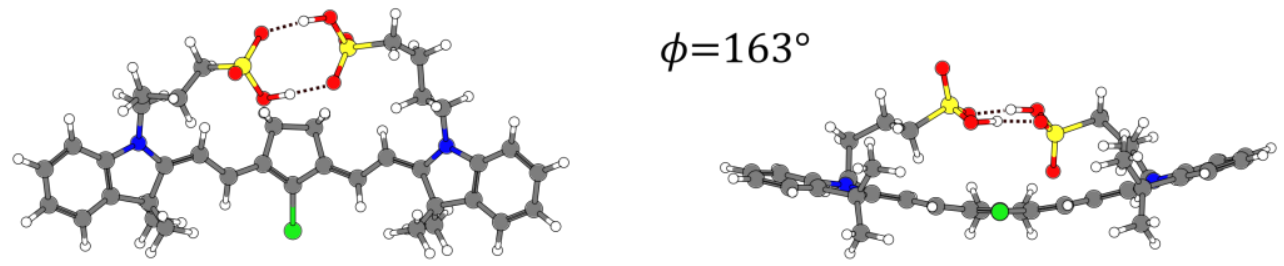

d) [IR806. $\mathrm{HNa}]^{+}:$756nm (up)/776nm (down)
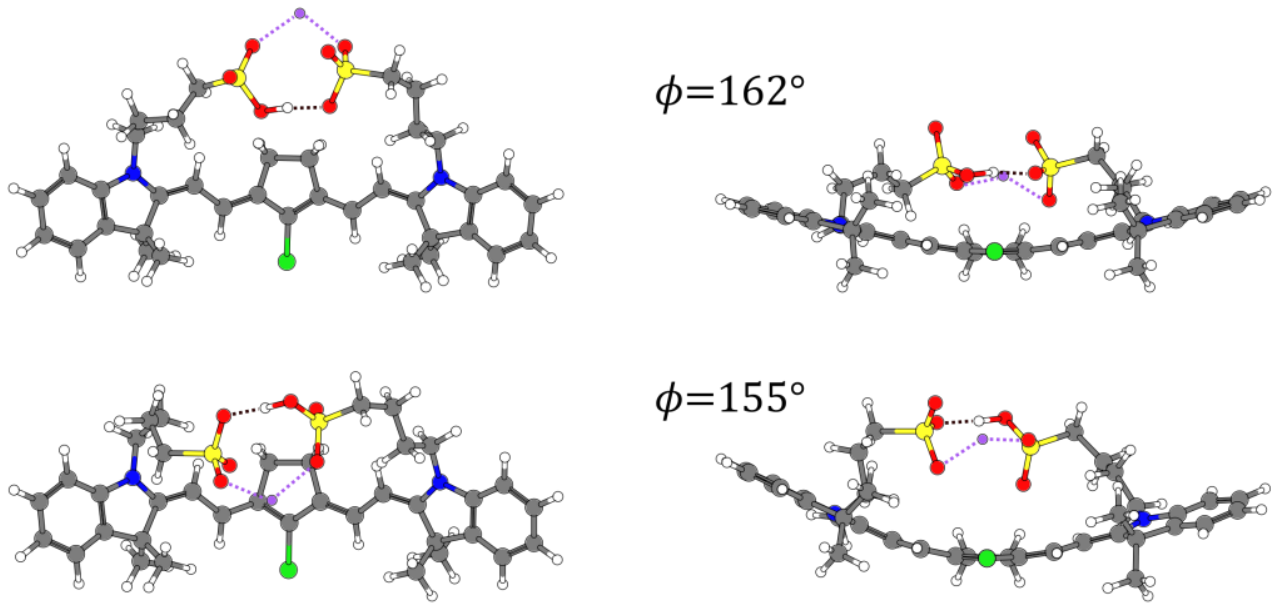

e) $[\text { IR806.2Na] }]^{+}: 778 \mathrm{~nm}$
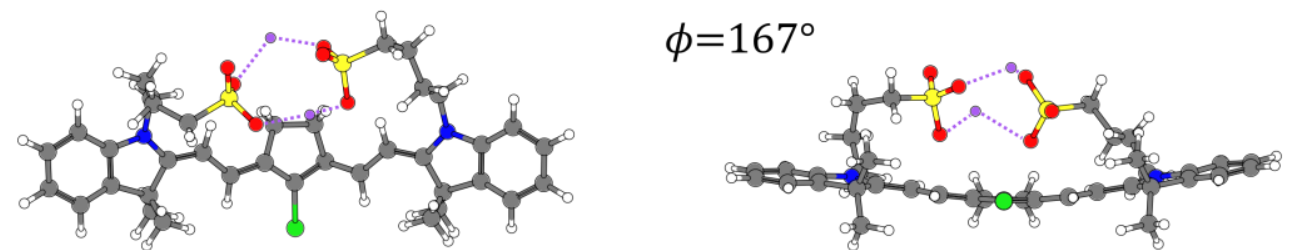

Figure S5. Optimized geometries of the five studied species, view from the top (left) and from the side (right). The dihedral angle of the polymethine chain is also shown. 


\section{Computation of the Absorption Wavelengths}

Following geometry optimization, the electronic structure was calculated at the CIS(D)/def2tzvp level in the ground state geometry, from which theoretical absorption wavelengths can be derived. Different DFT functionals and basis functions, in both DFT and CIS(D) calculations, were tested for [IR797] $]^{+}$, and the benchmarking result is shown in Table S1. For all the DFT methods used here, the difference with the experimental maximum absorption wavelength is always very high $(>130 \mathrm{~nm})$, independently of the functional or basis set, as observed in the literature ${ }^{2}$. Wavefunction methods including correlation greatly improved the comparison with the experiment. The very low difference (less than $20 \mathrm{~nm}$ ) enables us to interpret the experimental tendency accurately using the CIS(D) computations.

\begin{tabular}{|c|c|c|}
\hline Method & $\lambda_{\text {theo }}(\mathrm{nm})$ & $\left(\lambda_{\text {exp }}-\lambda_{\text {theo }}\right)(n m)$ \\
\hline B3LYP/6-311+G(2d,p) & 595 & 165 \\
\hline CAM-B3LYP/6-311+G(2d,p) & 588 & 172 \\
\hline$M 062 X / 6-311+G(2 d, p)$ & 595 & 165 \\
\hline$P B E / 6-311+G(2 d, p)$ & 621 & 139 \\
\hline$B L Y P / 6-311+G(2 d, p)$ & 624 & 136 \\
\hline$\omega B 97 X D / 6-311+G(2 d, p)$ & 590 & 170 \\
\hline B3LYP/def2-tzvp & 593 & 167 \\
\hline CAM-B3LYP/def2-tzvp & 585 & 175 \\
\hline M062X/def2-tzvp & 594 & 166 \\
\hline PBE/def2-tzvp & 620 & 140 \\
\hline BLYP/def2-tzvp & 622 & 138 \\
\hline$\omega B 97 X D /$ def2-tzvp & 588 & 172 \\
\hline CIS(D)/def2-svp & 736 & 24 \\
\hline $\mathrm{CIS}(\mathrm{D}) / \mathrm{ma}$-def2-svp & 744 & 16 \\
\hline $\mathrm{CIS}(\mathrm{D}) / \mathrm{def} 2-\mathrm{tzvp}$ & 743 & 17 \\
\hline
\end{tabular}

Table S1. Absorption wavelength of $[\text { IR797 }]^{+}$calculated with different methods, compared with the experimental absorption maximum $\left(\lambda_{\exp }=780 \mathrm{~nm}\right)$.

Following benchmarking on [IR797] ${ }^{+}$, our reference, we conducted the computations on the IR806 derivatives using the CIS(D) methodology at the def2-tzvp level, in the most stable geometries. The resulting wavelengths are displayed on Table 1 of the main text. We also extracted the absorption wavelengths of the conformations with "straight" substituents for the cationic derivatives of IR806, i.e. without folding to complex both cations (see for example Fig S4.b, showing both "folded" (left) and "straight" (right) conformations of $\left[\right.$ [R806.2Na] ${ }^{+}$). The result is in Table S2. For these "straight" conformations, the absorption wavelength remains roughly constant, similar to the one of [IR806] ${ }^{-}$. This is because adding counter cations to [IR806] in this conformation does not significantly influence the polymethine chromophore. To explain the observed tendency, we therefore need to consider the folding of the substituents, i.e. the most stable conformations. 


\begin{tabular}{cccccc}
\hline Parent ion & $\lambda_{\max }(\mathrm{nm})$ & $\begin{array}{c}\lambda_{\text {theo }} \\
\text { "folded"(nm) }\end{array}$ & $\begin{array}{c}\lambda_{\text {theo }} \\
\text { "straight"(nm) }\end{array}$ & $\begin{array}{c}\Delta \mathrm{E}(\mathrm{MP} 2) \\
(\mathrm{eV})\end{array}$ & $\begin{array}{c}\Delta \mathrm{E}(\mathrm{DFT}) \\
(\mathrm{eV})\end{array}$ \\
\hline [IR797] $^{+}$ & 760 & 743 & - & - & - \\
{$[\mathrm{IR} 806]^{-}$} & 780 & 761 & - & - & - \\
{$[\mathrm{IR} 806.2 \mathrm{H}]^{+}$} & 780 & 760 & 756 & 0.56 & 0.50 \\
{$[\mathrm{IR} 806 . \mathrm{HNa}]^{+}$} & 785 & $756 / 776$ & 750 & $0.73 / 0.84$ & $0.87 / 0.73$ \\
{$[\mathrm{IR} 806.2 \mathrm{Na}]^{+}$} & 790 & 778 & 757 & 1.37 & 1.32 \\
\hline
\end{tabular}

Table S2. Calculated absorption wavelength of the IR806 cationic derivatives for the "folded" (most stable) and "straight" conformations. $\Delta E=E$ ("straight")-E("folded") is the energy difference between these two conformations, calculated at the MP2/def2-tzvp or B3LYP/6$31+G *$ level.

\section{Electronic and Structural Analysis}

Following approaches from the literature ${ }^{3-6}$, we computed the central charge as well as Bond Length Alternation (BLA) for the different species. The central charge was calculated by Natural Population Analysis (NPA), applied to the central carbon atom indexed 0 in figure S6. BLA was deduced from the optimized geometries. Figure $\mathrm{S} 6$ shows examples of the bond lengths along the polymethine chain of [IR797] ${ }^{+}$and [IR806]', following the B3LYP geometry optimization. It can be seen that the bond lengths are not perfectly alternating around the mean value, partly due to the central ring and the central symmetry. For this reason, we computed the BLA as the average absolute difference between two consecutive $\mathrm{C}-\mathrm{C}$ bond lengths along the chromophore:

$$
B L A=\left\langle\left|d_{C C}(i+1)-d_{C C}(i)\right|\right\rangle
$$

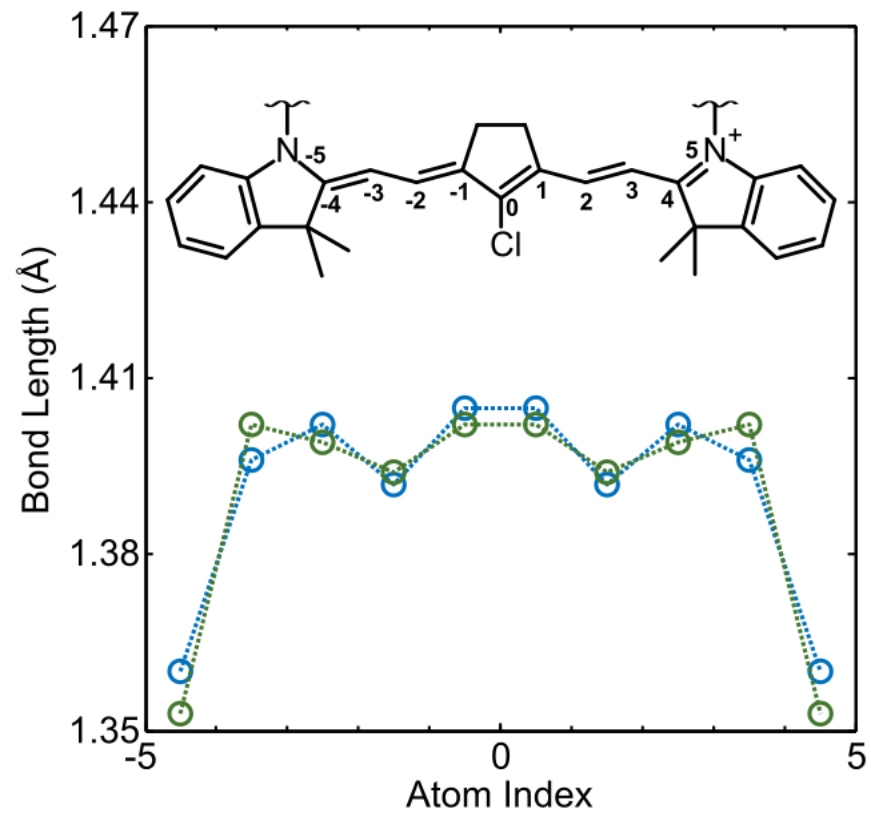

Figure S6. Bond lengths along the polymethine chain extracted from the computations, for [IR797] $^{+}$(blue) and [IR806] (green). As indicated on the figure, atoms are indexed from -5 to +5 between the two nitrogen atoms. 
To ensure that the BLA variations observed in this work are reliable, geometry optimization was also performed for [IR797] ${ }^{+}$using the Perdew, Burke and Ernzerhof (PBE $\alpha$ ) functional ${ }^{7}$, with $\alpha=0.456$. This functional was indeed shown to give satisfactory results in terms of BLA for cyanines ${ }^{8}$. Figure S7 reports the evolution of the bond length along the polymethine chain after geometry optimization using B3LYP and PBE $\alpha$ functionals. While the mean bond length slightly differs between the two functionals, the alternation along the backbone is very similar. The resulting BLA is thus, within the appreciable precision of the values, the same for the two methods, equal to $0.83 \mathrm{pm}$.

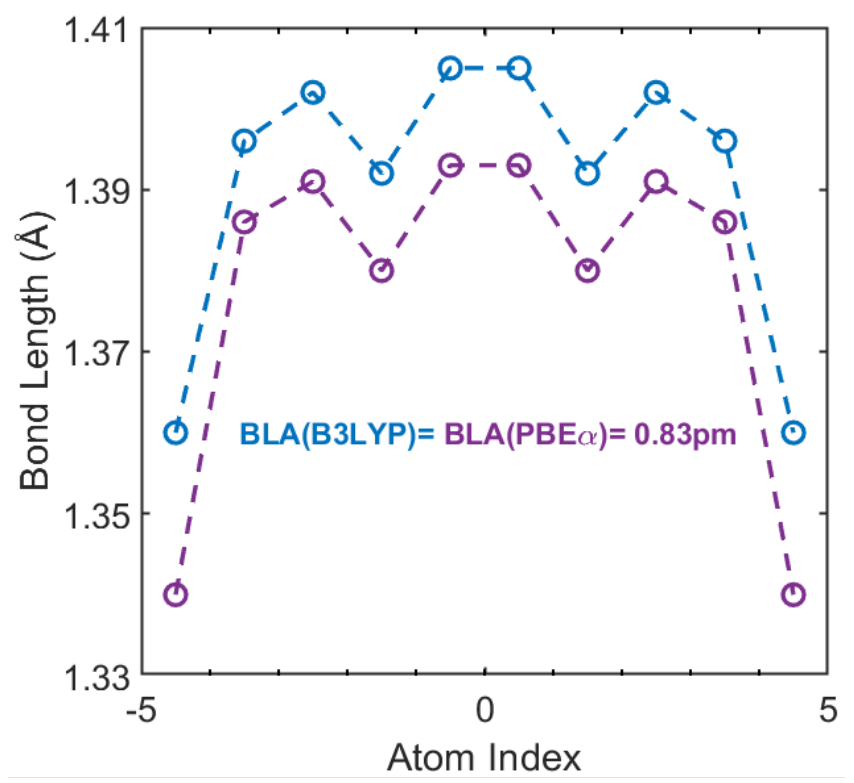

Figure S7. Bond lengths along the polymethine chain for $[I R 797]^{+}$, extracted from $B 3 L Y P$ geometry optimization (blue) and PBE $\alpha$ geometry optimization (purple).

While Fig 3.b of the main text shows the evolution of the BLA extracted from the most stable conformations of the studied molecular systems, figure S8 shows the extracted BLA for the conformations with "straight", unfolded substituents. For these conformations, the absorption wavelength decreases as the BLA increases. This is in accordance with the structure-properties relationship mentioned in the main text. 


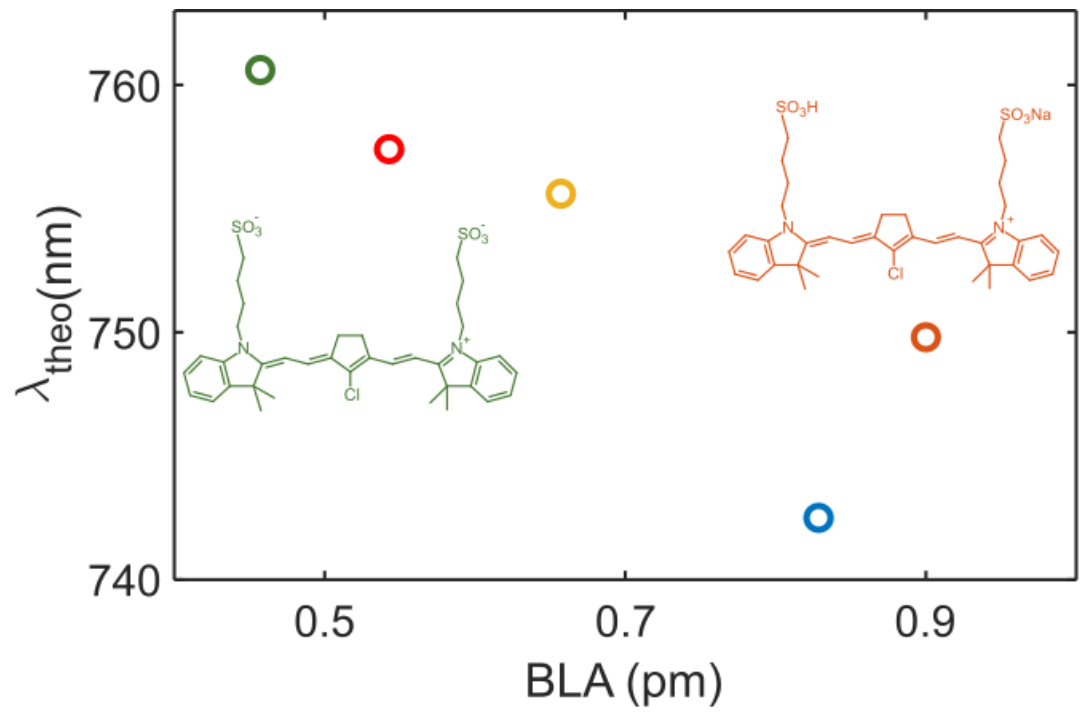

Figure S8. Correlation between the computed wavelength and the BLA for the studied species, where conformations with unfolded substituents have been considered for the cationic IR806 derivatives. Blue: [IR797] ${ }^{+}$; green: [IR806]'; yellow: [IR806.2H] ${ }^{+}$; orange: [IR806. $\mathrm{HNa}]^{+}$; red: [IR806.2Na] $]^{+}$

As described in the main text, the charge on the central carbon was taken as a diagnostic parameter, quantifying the weight of the bis-dipolar configuration. This parameter is indeed adapted to the present case, where gas phase folding occurs towards the center of the molecule. The bis-dipolar configuration is crucial in the determination of absorption properties for cyanines ${ }^{5}$, and decreasing its contribution generally leads to charge delocalization along the polymethine backbone. Figure S9 represents the charge alternation along the chain, calculated as the averaged absolute difference between two consecutive charges on the carbon atoms, for the different studied species, following the formula:

Charge Alternation $=\langle|\operatorname{Charge}(i+1)-\operatorname{Charge}(i)|\rangle$

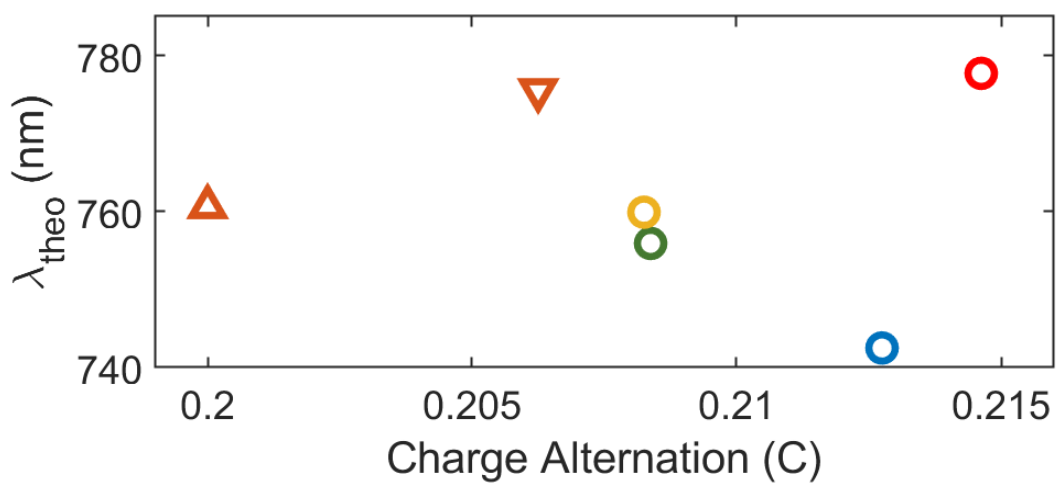

Figure 59. Correlation between the calculated absorption wavelength and the charge alternation along the carbon atoms, for the different species studied here. Blue: [IR797] ${ }^{+}$; green: [IR806] ; yellow: [IR806.2H] ${ }^{+}$; orange: [IR806. $\left.\mathrm{HNa}\right]^{+}$; red: [IR806.2Na] $]^{+}$. The orange upward-pointing triangle corresponds to the left geometry of [IR806. $\mathrm{HNa}]^{+}$on Figure 2 in main text, and the downward-pointing triangle to the right geometry. 
As can be seen, the evolution of charge alternation is as straightforward as for the central charge. This is because the central charge directly reflects the bis-dipolar configuration, and it is changed by the presence of the counter-cations. As counter-cations have an influence only at the center of the molecule, charge alternation is misleading, since it has to be chosen as a criterium for non-local constrains, as e.g. electric fields applied to the molecule. Choosing a diagnostic parameter that is adapted to the considered molecular system is thus compulsory to accurately predict absorption properties.

\section{References}

(1) Mills, E. A.; Regan, M. H.; Stanic, V.; Collings, P. J. Large Assembly Formation Via a TwoStep Process in a Chromonic Liquid Crystal. J. Phys. Chem. B 2012, 116, 13506-13515.

(2) Le Guennic, B.; Jacquemin, D. Taking Up the Cyanine Challenge with Quantum Tools. Acc. Chem. Res. 2015, 48, 530-537.

(3) Marder, S. R.; Gorman, C. B.; Meyers, F.; Perry, J. W.; Bourhill, G.; Brédas, J. L.; Pierce, B. M. A Unified Description of Linear and Nonlinear Polarization in Organic Polymethine Dyes. Science 1994, 265, 632-635.

(4) Bouit, P.-A.; Aronica, C.; Toupet, L.; Le Guennic, B.; Andraud, C.; Maury, O. Continuous Symmetry Breaking Induced by Ion Pairing Effect in Heptamethine Cyanine Dyes: beyond the Cyanine Limit. J. Am. Chem. Soc. 2010, 132, 4328-4335.

(5) Pascal, S.; Haefele, A.; Monnereau, C.; Charaf-Eddin, A.; Jacquemin, D.; Le Guennic, B.; Andraud, C.; Maury, O. Expanding the Polymethine Paradigm: Evidence for the Contribution of a Bis-dipolar Electronic Structure. J. Phys. Chem. A 2014, 118, 40384047.

(6) Gieseking, R. L.; Risko, C.; Brédas, J.-L. Distinguishing the Effects of Bond-Length Alternation versus Bond-Order Alternation on the Nonlinear Optical Properties of $\pi$ Conjugated Chromophores. J. Phys. Chem. Lett. 2015, 6, 2158-2162.

(7) Perdew, J. P.; Ernzerhof, M.; Burke, K. Rationale for Mixing Exact Exchange with Density Functional Approximations. J. Chem. Phys. 1996, 105, 9982-9985.

(8) Gieseking, R. L.; Ravva, M. K.; Coropceanu, V.; Brédas, J.-L. Benchmarking Density Functional Theory Approaches for the Description of Symmetry Breaking in Long Polymethine Dyes. J. Phys. Chem. C 2016, 120, 9975-9984. 